\title{
Correlation between autophagy levels in peripheral blood mononuclear cells and clinical parameters in patients with chronic obstructive pulmonary disease
}

\author{
YANJUN WU, BO XU, XIN HE, BO WU, YUNXIAO LI, GANGGANG YU, CHUNTING TAN and HAOYAN WANG \\ Department of Respiratory Medicine, Beijing Friendship Hospital, Capital Medical University, Beijing 100050, P.R. China
}

Received November 11, 2017; Accepted March 2, 2018

DOI: $10.3892 / \mathrm{mmr} .2018 .8831$

\begin{abstract}
Autophagy serves a role in the pathogenesis of chronic inflammatory diseases. The aim of the present study was to compare the autophagy levels in the peripheral blood mononuclear cells (PBMCs) of patients with chronic obstructive pulmonary disease (COPD) and healthy individuals and to assess the association between autophagy and the clinical parameters of COPD. Samples of peripheral blood from 20 patients with stable COPD and 20 healthy controls were collected. PBMCs were harvested using Ficoll density gradient centrifugation. Levels of the autophagy-associated proteins ubiquitin-binding protein p62 (p62), microtubule-associated proteins $1 \mathrm{~A} / 1 \mathrm{~B}$ light chain $3 \mathrm{~A}$ (LC3I/II) and beclin-1 in PBMCs were detected by western blotting. Enzyme-linked immunosorbent assay kits were used to detect the serum concentrations of interleukin (IL)-6, IL-8 and tumor necrosis factor (TNF)- $\alpha$. Associations between the levels of autophagy and forced expiratory volume in $1 \mathrm{sec} \%$ predicted $\left(\mathrm{FEV}_{1} \%\right)$ and pro-inflammatory factors were assessed. Western blotting demonstrated that the protein expression of p62 was decreased, but LC3II/I and beclin-1 levels increased in patients with COPD compared with healthy controls. Serum levels of IL-6, IL- 8 and TNF- $\alpha$ were increased in patients with COPD. The extent of PBMC autophagy was negatively correlated with $\mathrm{FEV}_{1} \%$ predicted, but positively correlated with levels of pro-inflammatory cytokines. The levels of autophagy in PBMCs in patients with COPD were increased and were negatively correlated with $\mathrm{FEV}_{1} \%$ predicted and positively correlated with circulating levels of pro-inflammatory cytokines. Autophagy may serve a role as a
\end{abstract}

Correspondence to: Professor Haoyan Wang or Dr Chunting Tan, Department of Respiratory Medicine, Beijing Friendship Hospital, Capital Medical University, 95 Yong An Road, Beijing 100050, P.R. China

E-mail: haoyanw@ccmu.edu.cn

E-mail: chuntingtan@126.com

Key words: autophagy, peripheral blood mononuclear cells, chronic obstructive pulmonary disease biomarker of the severity of COPD or as a therapeutic target for treatment of COPD.

\section{Introduction}

Chronic obstructive pulmonary disease (COPD) is a chronic inflammatory disease of the airways. It is characterized by progressive and incompletely reversible airflow limitation (1). COPD severely affects the quality of life of patients and results in an economic burden to them, their families, healthcare providers and society (2). Worldwide, COPD ranks as the third most common cause of death and the fifth most common cause of chronic disability $(1,2)$. Chronic cigarette smoking, exposure to noxious gases/particles and aging are the most common risk factors for the occurrence and development of COPD (3). However, in rural areas and in populations with low socioeconomic status, nutritional status and use of indoor biofuels are equally important risk factors $(3,4)$.

Inflammation and the inflammatory mediators released from immune cells accumulate in the lungs of patients with COPD. This action can contribute to the development of airway remodeling, which includes thickening of bronchial smooth muscle and deposition of the extracellular matrix $(5,6)$. Pulmonary inflammation can lead to airway stenosis, increased airway resistance and reduced lung function in patients with COPD $(5,6)$. Furthermore, there is increasing evidence indicating that chronic inflammation in patients with COPD is also a systemic inflammatory response $(7,8)$.

Autophagy is the regulated destructive mechanism of the cell that disassembles unnecessary or dysfunctional components. This physiological mechanism identified primarily in polymorphonuclear phagocytes and mononuclear phagocytes, is increased during chronic inflammation. However, excessive activation of autophagy has been demonstrated to promote apoptosis and inflammation (9). Previous studies indicated that autophagy is involved in the pathogenesis of COPD (9-11). Using a mouse model of COPD induced by smoke inhalation, the authors of the present study previously demonstrated that autophagy was associated with inflammation as well as the development of emphysema and airway resistance (12). The authors have also previously demonstrated that expression of autophagy-associated proteins increased in a human bronchial epithelial cell line (Beas-2B) treated with cigarette smoke 
condensate and that 14,15-epoxyeicosatrienoic can inhibit the inflammatory response through suppression of autophagy induced by cigarette smoke (13). Autophagy involves a pathway of cell metabolism that leads to phagocytic cell engulfing its own cytoplasmic proteins or organelles which form vesicles that fuse with lysosomes to create autophagosomes, which can be observed by electron microscopy (14). Autophagy can be classified into macro-autophagy, micro-autophagy and molecular chaperone-mediated autophagy (15).

To the best of our knowledge, no studies have evaluated autophagy and expression of pro-inflammatory cytokines in the peripheral blood mononuclear cells (PBMCs) of patients with COPD. The present study compared the levels of autophagy of PBMCs in patients with COPD with those of healthy controls and assessed the association between autophagy and the clinical parameters of COPD.

\section{Materials and methods}

Ethical approval of the study protocol. The study protocol complied with the Declaration of Helsinki and Good Clinical Practice guidelines. The study protocol was approved by the Ethics Committee of Beijing Friendship Hospital (approval no. 2017-P2-014-01). All individuals who participated in this study provided written informed consent.

Chemicals and reagents. Ficoll separation medium was purchased from Tianjin HaoYang Biological Products Technology Co., Ltd. (Tianjin, China). Radioimmunorecipitation assay (RIPA) lysis buffer was obtained from Beyotime Institute of Biotechnology (Shanghai, China). Bicinchoninic acid (BCA) Protein Assay kit, enhanced chemiluminescent (ECL) substrate and PageRuler ${ }^{\mathrm{TM}}$ Prestained Protein Ladder were purchased from Thermo Fisher Scientific, Inc. (Waltham, MA, USA). Nitrocellulose membranes were obtained from Applygen Technologies, Inc. (Beijing, China). A ChemiDoc ${ }^{\mathrm{TM}}$ MP system for detecting the antibody-antigen reactivity of western blotting was purchased from Bio-Rad Laboratories, Inc. (Hercules, CA, USA). The clinical spirometer used purchased from Jaeger; Vyaire Medical Inc. (Würzburg, Germany). Enzyme-linked immunosorbent assay (ELISA) kits for human interleukin (IL)-6 (cat. no. EH08-96), IL-8 (cat. no. EH07-96) and tumor necrosis factor (TNF)- $\alpha$ (cat. no. EH02-96) were obtained from Beijing Bio-Biotech Co., Ltd. (Beijing, China). The primary antibodies against microtubule-associated proteins 1A/1B light chain 3A (LC3I/II; cat. no. ab62721; 1:1,000), ubiquitin-binding protein p62 (p62; cat. no. ab56416; 1:1,000) and beclin-1 (cat. no. ab207612, 1:2,000) were purchased from Abcam (Cambridge, MA, USA). GAPDH (cat. no. 60004-1-lg; 1:10,000) was obtained from ProteinTech Group, Inc. (Chicago, IL, USA). Peroxidase-conjugated goat anti-mouse (cat. no. ZB-2305; 1:5,000) and goat anti-rabbit (cat. no. ZB-2301; 1:5,000) secondary antibodies were purchased from OriGene Technologies, Inc. (Beijing, China).

Recruitment of patients and healthy controls. A control group of healthy people ( $n=20$; male) and patients with stable COPD ( $n=20$; male) were recruited at the Beijing Friendship Hospital, Capital Medical University (Beijing, China) between February and July 2017.
Inclusion criteria for the control group were healthy adults without chronic respiratory disease, cancer, hematologic disease, hypertension, arrhythmia, ischemic heart disease or diabetes mellitus. Patients with COPD were included if their disease was stable. Exclusion criteria were diagnosis of COPD with other chronic respiratory diseases, including asthma, bronchiectasis, interstitial lung disease. The study population was free from ischemic heart disease, kidney disease, cancer or autoimmune disease. Lung-function tests were undertaken by a single spirometry technician experienced in carrying out such tests. The diagnostic criteria for stable COPD were in accordance with current recommendations set by the Global Initiative for Chronic Obstructive Lung Disease (16).

Isolation of PBMCs. Ethylenediamine tetraacetic acid Vacutainer $^{\mathrm{TM}}$ tubes (BD Biosciences, Franklin Lakes, NJ, USA) were used to collect venous blood samples $(10 \mathrm{ml})$ from fasting participants in the early morning. Aliquots of the supernatant were collected by centrifugation at $800 \mathrm{x} \mathrm{g}$ and $25^{\circ} \mathrm{C}$ for $15 \mathrm{~min}$ and subsequently stored at $-80^{\circ} \mathrm{C}$. The remaining blood was mixed and added slowly dropwise to a centrifuge tube containing $10 \mathrm{ml}$ of Ficoll separation medium. PBMCs were isolated according to manufacturer's protocol and stored at $-80^{\circ} \mathrm{C}$.

Western blotting. PBMCs were lysed with RIPA lysis buffer and protein concentrations determined with the BCA Protein Assay kit according to the manufacturer's protocol. Subsequently, $20 \mu \mathrm{g}$ of protein/well were separated using $12 \%$ SDS-PAGE. The proteins were subsequently transferred to nitrocellulose membranes at $300 \mathrm{~mA}$ for $60 \mathrm{~min}$. The nitrocellulose membranes were blocked with Tris-buffered saline containing $0.1 \%$ Tween 20 (TBST) and 5\% non-fat milk powder for $2 \mathrm{~h}$ at room temperature. The nitrocellulose membranes were incubated with primary antibodies overnight at $4^{\circ} \mathrm{C}$ on a shaker set at a slow speed. The nitrocellulose membranes were washed thrice with TBST and incubated with secondary antibodies for $1 \mathrm{~h}$ at room temperature. After washing thrice, ECL substrate was added to the nitrocellulose membrane. The signal was detected using Image $\mathrm{Lab}^{\mathrm{TM}}$ software (version 5.1; Bio-Rad Laboratories, Inc.). Band density was quantified with ImageJ software (version 1.51; National Institutes of Health, Bethesda, MD, USA).

ELISA. Serum levels of IL-6, IL-8 and TNF- $\alpha$ were measured by ELISA according to manufacturer's protocol. All samples were measured in duplicate.

Statistical analyses. All statistical analyses were carried out using Prism 5 (GraphPad Software, Inc., La Jolla, CA, USA). Normally distributed data are presented as the mean \pm standard deviation. The statistical difference of the levels of autophagy and pro-inflammatory cytokines between patients with COPD and healthy controls was performed using the independent sample Student's t-test. Correlation between the levels of autophagy, serum levels of pro-inflammatory cytokines and pulmonary function was determined using Pearson's or Spearman's tests, for normally and not normally distributed data, respectively. $\mathrm{P}<0.05$ was considered to indicate a statistically significant difference. 


\section{Results}

Characterization of the study cohort. The study cohort was divided into 20 healthy controls and 20 patients with COPD. Clinical characteristics of this cohort, including age, body mass index, blood tests and lung function parameters are summarized in Table I. Neutrophil counts and lung function were significantly different between patients with COPD and healthy subjects.

Autophagy levels of PBMCs were increased in patients with $C O P D$. Western blotting (Fig. 1) demonstrated that expression of the autophagy-associated protein p62 was reduced compared with the control group (Fig. 1B; $\mathrm{P}=0.0011$ ). Furthermore, beclin-1 expression (Fig. 1C; $\mathrm{P}<0.0001$ ) and LC3II/I ratio (Fig. 1D; $\mathrm{P}=0.0249$ ) were increased compared with the control group.

Cytokine levels were increased in patients with COPD. ELISA results (Fig. 2) exhibited significantly increased serum levels (in pg/ml) of IL-6 (11.96 \pm 3.46 vs. $2.70 \pm 1.72 ; \mathrm{P}<0.0001$; Fig. 2A), IL-8 (20.38 \pm 18.44 vs. $6.0 \pm 4.08$; $\mathrm{P}=0.0016$; Fig. $2 \mathrm{~B})$, and TNF- $\alpha(29.5 \pm 28.18$ vs. $11.08 \pm 12.07 ; \mathrm{P}=0.0106$; Fig. $2 \mathrm{C})$ in patients with COPD compared with the control group.

Levels of pro-inflammatory cytokines were associated with autophagy levels in patients with COPD. Correlation analyses (Fig. 3) demonstrated that levels of IL-6 ( $\mathrm{r}=-0.4675 ; \mathrm{P}=0.0377$; Fig. 3A), IL-8 ( $\mathrm{r}=-0.5366 ; \mathrm{P}=0.0147$; Fig. 3B) and TNF- $\alpha$ $(\mathrm{r}=-0.6065$; $\mathrm{P}=0.0046$; Fig. $3 \mathrm{C}$ ) were negatively correlated with p62/GAPDH levels. Levels of IL-6 ( $\mathrm{r}=0.5094 ; \mathrm{P}=0.0218$; Fig. 3D), IL-8 (r=0.4841; $\mathrm{P}=0.0305$; Fig. 3E) and TNF- $\alpha$ $(\mathrm{r}=0.5454 ; \mathrm{P}=0.0129$; Fig. $3 \mathrm{~F})$ were positively correlated with beclin-1/GAPDH levels. Levels of IL-6 ( $r=0.6344$; $\mathrm{P}=0.0027$; Fig. 3G), IL-8 (r=0.5847; P=0.0068; Fig. 3H) and TNF- $\alpha$ $(\mathrm{r}=0.5818 ; \mathrm{P}=0.0071$; Fig. $3 \mathrm{I})$ were positively correlated with LC3II/I levels.

COPD severity according to lung function was correlated with autophagy. Correlation analyses (Fig. 4) demonstrated that forced expiratory volume in $1 \mathrm{sec} \%$ predicted $\left(\mathrm{FEV}_{1} \%\right.$ predicted) was positively correlated with p62/GAPDH levels in patients with COPD ( $\mathrm{r}=0.6702 ; \mathrm{P}=0.0012$; Fig. 4A). $\mathrm{FEV}_{1} \%$ predicted was negatively correlated with beclin-1/GAPDH levels ( $\mathrm{r}=-0.5306 ; \mathrm{P}=0.0161$; Fig. 4B) and with LC3II/I levels $(\mathrm{r}=-0.5967, \mathrm{P}=0.0055$; Fig. 4C).

\section{Discussion}

COPD is a public health problem. However, at present, the pathogenesis and therapeutic targets of COPD are not completely elucidated. Autophagy is common in eukaryotic cells. Increasingly sophisticated research on the role of autophagy has revealed that autophagy and COPD are closely associated (13,17-20).

To the best of the authors' knowledge, the present study is the first to demonstrate that levels of autophagy in PBMCs in patients with COPD were increased compared with normal controls and that autophagy levels were negatively correlated with $\mathrm{FEV}_{1} \%$ predicted, but positively correlated with circulating levels of pro-inflammatory cytokines.
Table I. Clinical characteristics of the study cohort.

\begin{tabular}{lrcr}
\hline Characteristic & \multicolumn{1}{c}{$\begin{array}{c}\text { Controls } \\
(\mathrm{n}=20)\end{array}$} & $\begin{array}{c}\text { Patients with } \\
\text { COPD }(\mathrm{n}=20)\end{array}$ & P-value \\
\hline Age (years) & $67.05 \pm 8.96$ & $62.25 \pm 5.30$ & 0.076 \\
Height $(\mathrm{cm})$ & $169.05 \pm 5.86$ & $168.5 \pm 5.38$ & 0.576 \\
Weight $(\mathrm{kg})$ & $70.0 \pm 6.59$ & $68.4 \pm 9.76$ & 0.307 \\
BMI $\left(\mathrm{kg} / \mathrm{m}^{2}\right)$ & $24.49 \pm 1.87$ & $24.04 \pm 2.69$ & 0.467 \\
WBC $\left(\mathrm{x} 10^{9} / 1\right)$ & $6.88 \pm 1.86$ & $7.08 \pm 1.76$ & 0.739 \\
NEU $(\%)$ & $61.62 \pm 9.20$ & $68.54 \pm 8.09$ & 0.018 \\
$\mathrm{FEV}_{1}(\%)$ & $98.02 \pm 3.71$ & $43.41 \pm 14.51$ & $<0.01$ \\
$\mathrm{FEV}_{1} / \mathrm{FVC}(\%)$ & $88.05 \pm 5.19$ & $59.98 \pm 8.40$ & $<0.01$
\end{tabular}

COPD, chronic obstructive pulmonary disease; BMI, body mass index; WBC, white blood cell; Neu \%, percentage of neutrophils; $\mathrm{FEV}_{1}$, forced expiratory volume in $1 \mathrm{sec} ; \mathrm{FVC}$, forced vital capacity. Data are presented as the mean \pm standard deviation. $\mathrm{P}<0.05$ was considered to indicate a statistically significant difference.

Exposure to cigarette smoke is the primary cause of COPD (13). Furlong et al (17) demonstrated that exposure to cigarette smoke can trigger an autophagic cascade by activating the adenosine monophosphate-activated protein kinase pathway in mouse ovaries. Gannon et al (18) demonstrated that, in murine granulosa cells induced by cigarette-smoke exposure, the levels of autophagy increased and mitochondrial dynamics were impaired. Chen et al (19) demonstrated that autophagy is involved in inflammation and that excess mucus production is induced by ultrafine particles in the airway epithelium. Zhou et al (20) demonstrated that autophagy serves an essential part in bronchial mucus production induced by cigarette smoke by regulating MUC5AC expression. The studies of Hussain and Sandri (21) and Plant et al (22) demonstrated that autophagy can affect the bronchial-muscle function of patients with COPD. The pathophysiological mechanisms involved in COPD, including oxidative stress and inflammation, are associated with autophagy (14,23-25). Therefore, autophagy serves a role in the pathogenesis of COPD. The results of our study support the current view that autophagy is involved in COPD.

In the present study, levels of LC3II/I were evaluated since in the process of autophagy, the characteristic microtubule-associated protein light chain LC3-I is converted to LC3-II and, therefore, LC3II/I levels are expected to increase $(26,27)$. Beclin-1 is an autophagy-mediated protein and its expression is known to increase during autophagy (28). p62 is an intracellular and multifunctional protein that contains a variety of protein domains and serves a role in signal transduction and pathogenesis of multiple diseases, including COPD (29-32). As a link between LC3 and polyubiquitinated proteins, p62 can transport damaged organelles or proteins into autophagosomes through the ubiquitin signaling pathway. Eventually, p62 and its substrates are degraded together in autophagosomes. Therefore, p62 levels are negatively correlated with autophagy levels, which can be used as a marker of autophagy (33). In the present study, p62 levels were reduced and those of LC3II/I and beclin-1 increased, in patients with COPD, compared with healthy controls. 
A

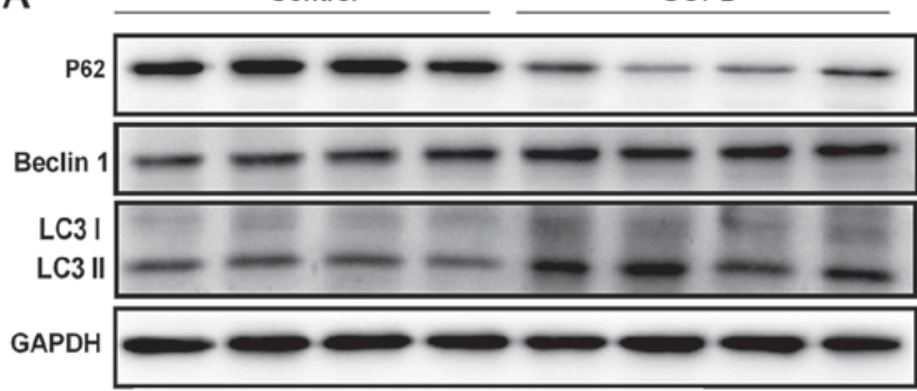

C

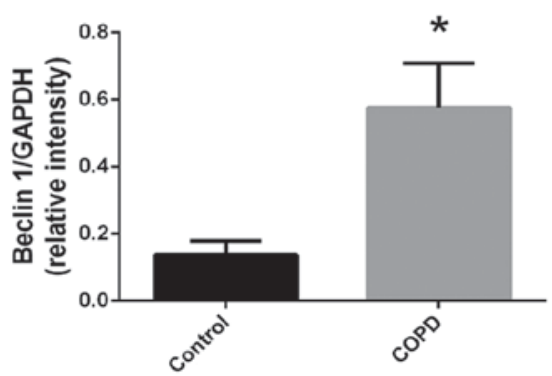

B

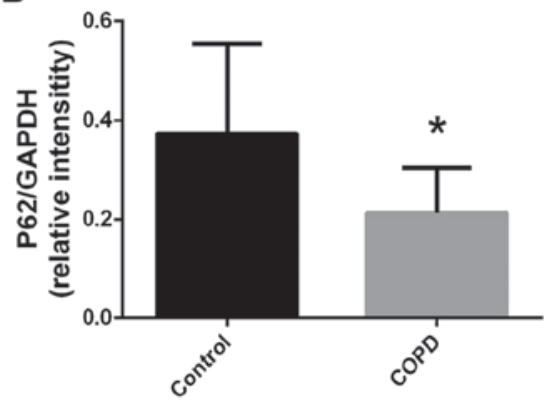

D

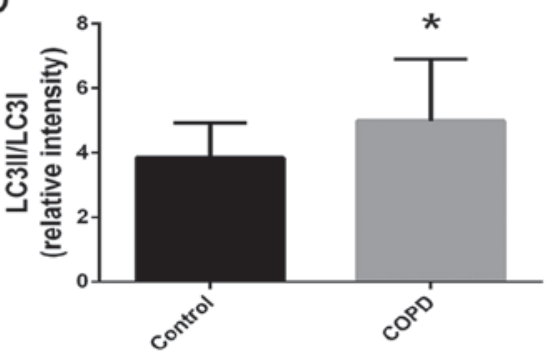

Figure 1. Autophagy levels of peripheral blood mononuclear cells in patients with COPD and healthy controls. (A) Western blot analysis of protein expression. Levels of the autophagy-associated proteins (B) p62, (C) beclin-1 and (D) LC3II/I were quantified. GAPDH was used as a loading control. Data are presented as the mean \pm standard deviation from three independent experiments. "P<0.05 vs. the control group. p62, ubiquitin-binding protein p62; LC3II/I, microtubule-associated proteins 1A/1B light chain 3A; COPD, chronic obstructive pulmonary disease.
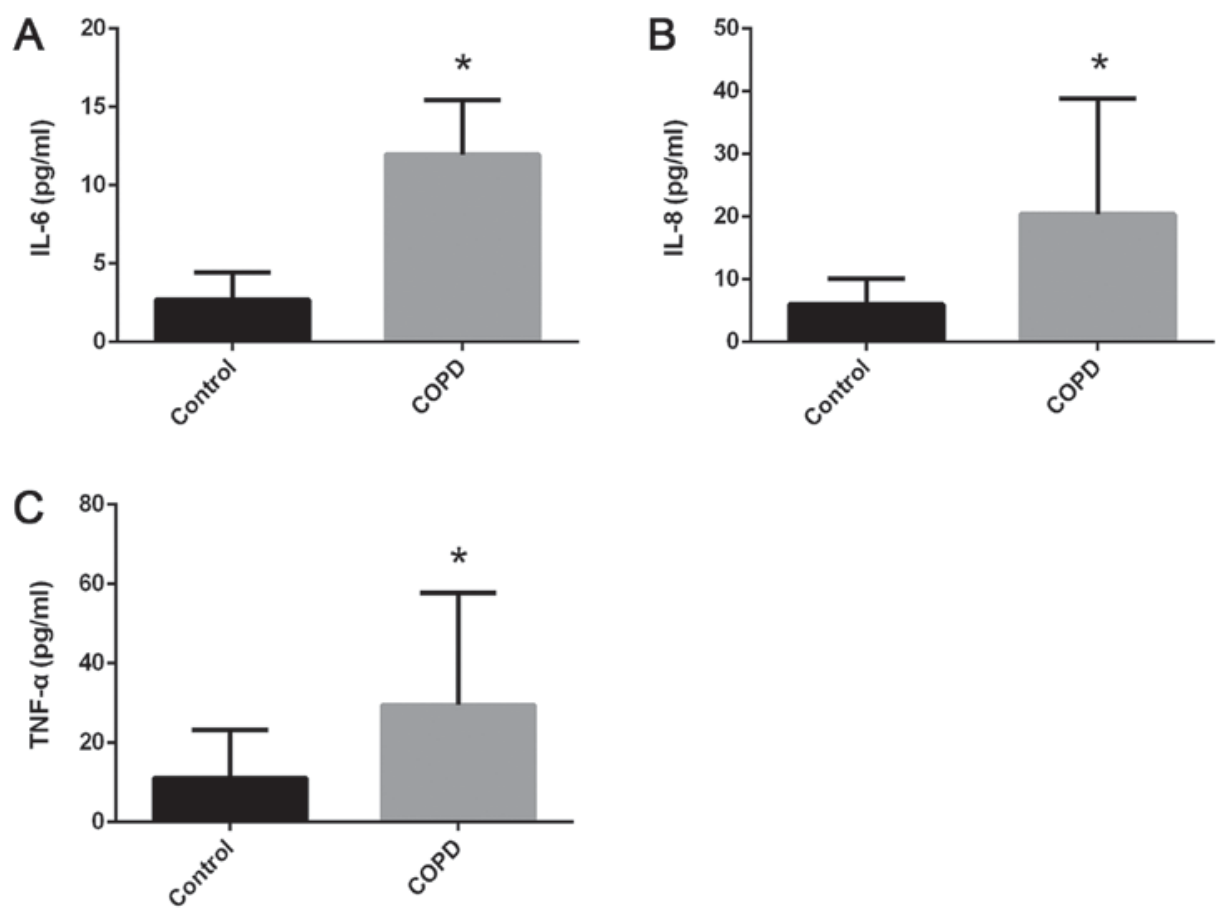

Figure 2. Serum levels of IL-6, IL-8 and tumor necrosis factor (TNF)- $\alpha$ in patients with COPD and healthy controls. The serum levels of (A) IL-6, (B) IL-8 and (C) TNF- $\alpha$ were significantly higher in patients with COPD compared with healthy controls. Data are presented as the mean \pm standard deviation from three independent experiments. "P $<0.05$ vs. the control group. IL, interleukin; TNF- $\alpha$, tumor necrosis factor- $\alpha$; COPD, chronic obstructive pulmonary disease.

The present study demonstrated that levels of the pro-inflammatory cytokines IL-6, IL-8 and TNF- $\alpha$ in the serum of patients with COPD were increased and were correlated with autophagy levels. The pathophysiological processes of inflammation, cytokine production and autophagy are involved in airway remodeling in COPD (34). One study demonstrated that the detection of IL-6 levels could be used to predict the frequency of future COPD exacerbations (35). The aforementioned study supports the view that inhibition of inflammation induced by inhibition of the production of pro-inflammatory mediators may prevent/limit the pulmonary parenchymal, vascular and airway alterations that result in clinical manifestations of COPD $(35,36)$. 

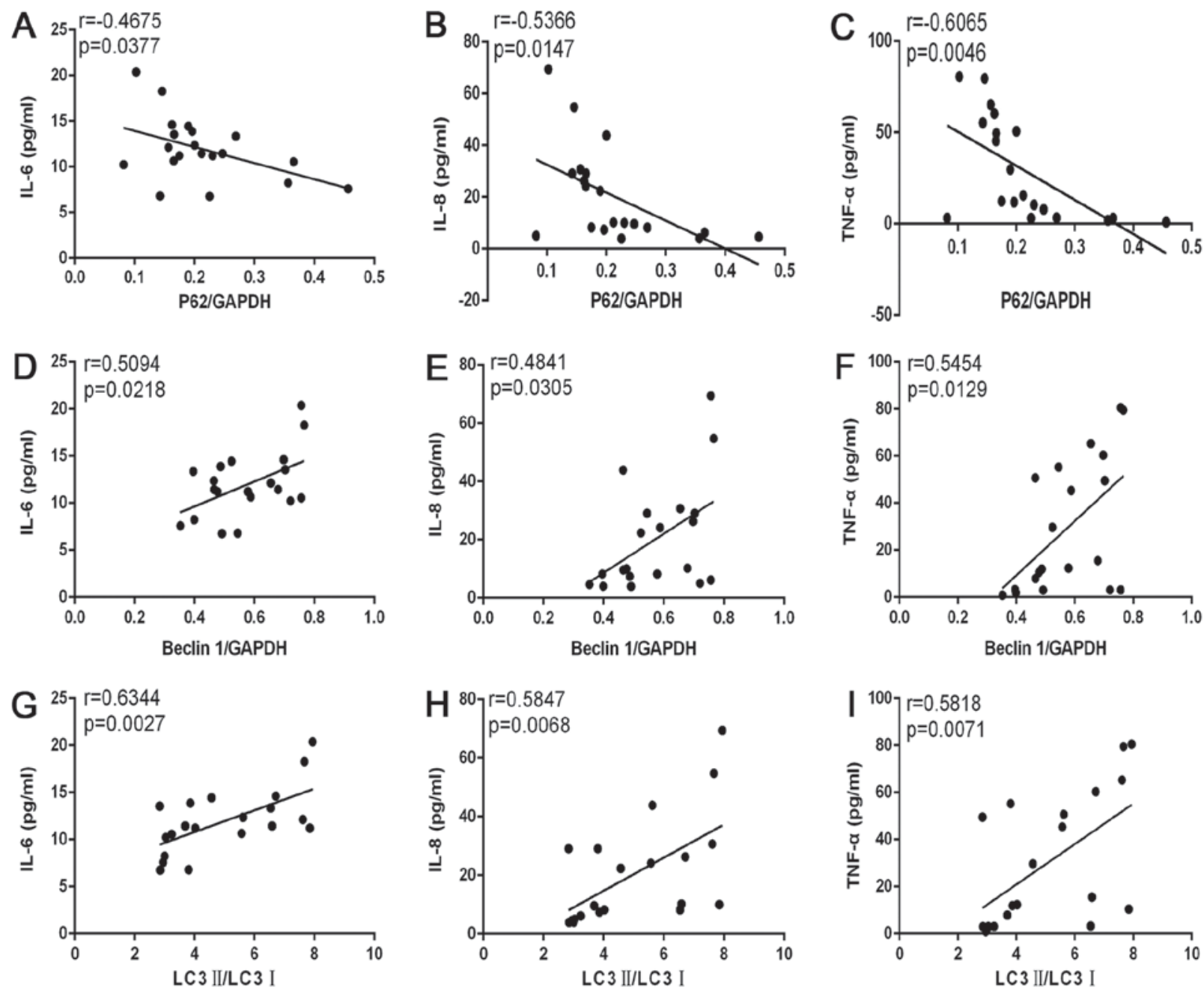

Figure 3. The association between inflammatory factors and autophagy levels in the PBMCs of patients with COPD. (A) Correlation between serum levels of IL-6 and p62/GAPDH levels in the PBMCs of patients with COPD. (B) Correlation between serum levels of IL-8 and p62/GAPDH levels in the PBMCs of patients with COPD. (C) Correlation between serum levels of TNF- $\alpha$ and p62/GAPDH levels in the PBMCs of patients with COPD. (D) Correlation between serum levels of IL-6 and beclin-1/GAPDH levels in the PBMCs of patients with COPD. (E) Correlation between serum levels of IL-8 and beclin-1/GAPDH levels in the PBMCs of patients with COPD. (F) Correlation between serum levels of TNF- $\alpha$ and beclin-1/GAPDH levels in the PBMCs of patients with COPD. (G) Correlation between serum levels of IL-6 and LC3II/I levels in the PBMCs of patients with COPD. (H) Correlation between serum levels of IL-8 and LC3II/I levels in the PBMCs of patients with COPD. (I) Correlation between serum levels of TNF- $\alpha$ and LC3II/I levels in the PBMCs of patients with COPD. $\mathrm{P}<0.05$ was considered to indicate a statistically significant difference. IL, interleukin; TNF- $\alpha$, tumor necrosis factor- $\alpha$; PBMC, peripheral blood mononuclear cell; COPD, chronic obstructive pulmonary disease.

Studies have demonstrated that autophagy may serve different roles in different diseases, including metabolic disease, neurodegenerative disease, infections and immunologic disease (37-39). Vij et al (40) demonstrated that cigarette smoke-induced impairment of autophagy accelerates lung aging. Li et al (41) reported that silymarin, similar to our previous study focusing on 14,15-epoxyeicosatrienoic acid (13), attenuates cigarette smoke extract-induced inflammation via inhibition of autophagy. The above studies, together with the present study indicating that autophagy levels of PBMCs in patients with COPD were positively correlated with circulating levels of inflammatory cytokines, suggest that autophagy may regulate the inflammatory response in COPD through different pathways.

The present study also demonstrated that the $\mathrm{FEV}_{1} \%$ predicted was negatively correlated with levels of autophagy in PBMCs of patients with COPD. This result raises the possibility that autophagy may be used as a biomarker to evaluate severity of COPD. There is an association between autophagy, inflammation and lung function, and a high level of autophagy can activate the inflammatory response, and chronic inflammation can lead to airway remodeling and accelerate reductions in lung function. By contrast, long periods of low levels of oxygen and hypercapnia due to airway obstruction can aggravate inflammation. The above phenomena can influence each other $(42,43)$.

The present study had three main limitations. The study cohort was entirely male and the medication history of patients was not studied in detail. Furthermore, according to the $\mathrm{FEV}_{1} \%$ predicted value, the study cohort contained only six patients with moderate airflow limitation and no patients with mild airflow limitation; the remaining patients had severe and very severe airflow limitation.

In conclusion, the present study demonstrated that autophagy levels in PBMCs in patients with COPD were increased and were correlated with $\mathrm{FEV}_{1} \%$ predicted values 

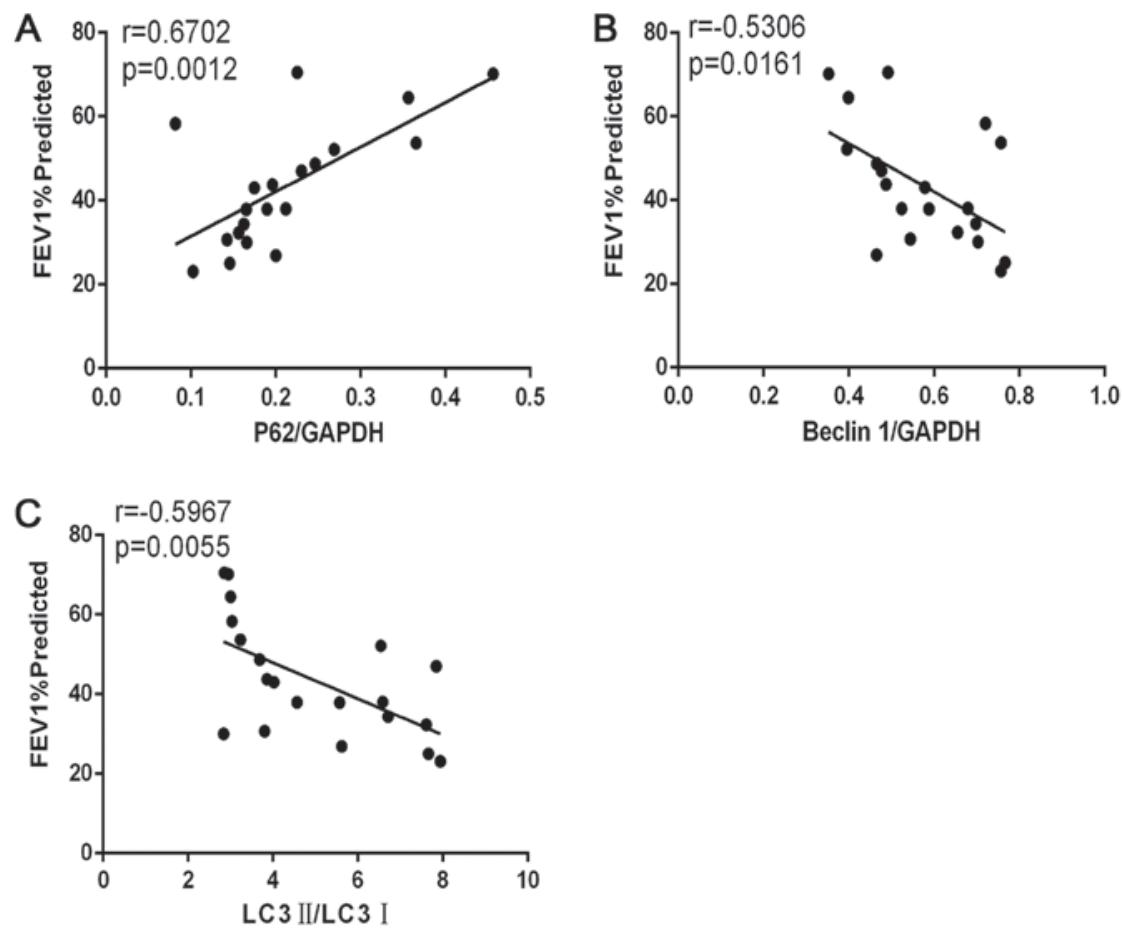

Figure 4. The association between $\mathrm{FEV}_{1} \%$ predicted and autophagy levels in the peripheral blood mononuclear cells of patients with COPD. (A) The association between $\mathrm{FEV}_{1} \%$ predicted and $\mathrm{p} 62 / \mathrm{GAPDH}$ levels in the PBMCs of patients with COPD. (B) The association between $\mathrm{FEV}_{1} \%$ predicted and beclin-1/GAPDH levels in the PBMCs of patients with COPD. (C) The association between FEV \% predicted and LC3II/I levels in the PBMCs of patients with COPD. $\mathrm{P}<0.05$ was considered to indicate a statistically significant difference. PBMC, peripheral blood mononuclear cell; COPD, chronic obstructive pulmonary disease; 62 , ubiquitin-binding protein $\mathrm{p} 62 ; \mathrm{FEV} \%$ predicted; forced expiratory volume in 1 sec predicted; LC3II/I, microtubule-associated proteins $1 \mathrm{~A} / 1 \mathrm{~B}$ light chain $3 \mathrm{~A}$.

and circulating levels of pro-inflammatory cytokines. Autophagy may serve a role as a biomarker of severity or as a therapeutic target for treatment of COPD.

\section{Acknowledgements}

Not applicable.

\section{Funding}

The present study was supported by grants from the Basic-Clinical Cooperation Program of Capital Medical University (grant no. 17JL49), National Natural Science Foundation of China (grant no. 81700038), Beijing Municipal Natural Science Foundation (grant nos. 7142046 and 7164250) and the Capital Health Research and Development of Special (grant no. 2018-2-2024).

\section{Availability of data and materials}

The datasets used and/or analyzed during the current study are available from the corresponding author on reasonable request.

\section{Authors' contributions}

CT and HW conceived and designed the study. GY and CT provided administrative support. BW and YL provided the study materials and/or subjects. YW and BX collected and assembled the data. XH, BW, YL and BX provided technical support, analyzed and interpreted the data. YW and GY were involved in drafting the manuscript. CT and HW critically revised the manuscript. All authors read and approved the final manuscript.

\section{Ethics approval and consent to participate}

The study protocol complied with the Declaration of Helsinki and Good Clinical Practice guidelines. The study protocol was approved by the Ethics Committee of Beijing Friendship Hospital (2017-P2-014-01). All individuals who participated in this study provided written informed consent.

\section{Consent for publication}

Written informed consent was obtained from all individuals who participated in this study.

\section{Competing interests}

The authors declare that they have no competing interests.

\section{References}

1. Barnes PJ: Senescence in COPD and its comorbidities. Annu Rev Physiol 79: 517-539, 2017.

2. Chun P: Role of sirtuins in chronic obstructive pulmonary disease. Arch Pharm Res 38: 1-10, 2015.

3. Burney P, Kato B, Janson C, Mannino D, Studnicka M, Tan W, Bateman E, Koçabas A, Vollmer WM, Gislason T, et al: Chronic obstructive pulmonary disease mortality and prevalence: The associations with smoking and poverty: A BOLD analysis-authors' reply. Thorax 69: 869-870, 2014. 
4. Petrusca DN, Van Demark M, Gu Y, Justice MJ, Rogozea A, Hubbard WC and Petrache I: Smoking exposure induces human lung endothelial cell adaptation to apoptotic stress. Am J Respir Cell Mol Biol 50: 513-325, 2014

5. Sun J, Bao J, Shi Y, Zhang B, Yuan L, Li J, Zhang L, Sun M, Zhang L and Sun W: Effect of simvastatin on MMPs and TIMPs in cigarette smoke-induced rat COPD model. Int J Chron Obstruct Pulmon Dis 12: 717-724, 2017.

6. Zou Y, Chen X, Liu J, Zhou DB, Kuang X, Xiao J, Yu Q, Lu X, Li W, Xie B and Chen Q: Serum IL-1 $\beta$ and IL-17 levels in patients with COPD: Associations with clinical parameters. Int J Chron Obstruct Pulmon Dis 12: 1247-1254, 2017.

7. Gea J, Pascual S, Casadevall C, Orozco-Levi M and Barreiro E: Muscle dysfunction in chronic obstructive pulmonary disease: Update on causes and biological findings. J Thorac Dis 7: E418-E438, 2015.

8. Kawayama T, Kinoshita T, Matsunaga K, Kobayashi A, Hayamizu T, Johnson M and Hoshino T: Responsiveness of blood and sputum inflammatory cells in Japanese COPD patients, non-COPD smoking controls, and non-COPD nonsmoking controls. Int J Chron Obstruct Pulmon Dis 11: 295-303, 2016

9. Ryter SW, Lam HC, Chen ZH and Choi AM: Deadly triplex: Smoke, autophagy and apoptosis. Autophagy 7: 436-437, 2011.

10. Ryter SW, Lee SJ and Choi AM: Autophagy in cigarette smoke-induced chronic obstructive pulmonary disease. Expert Rev Respir Med 4: 573-584, 2010.

11. Ryter SW, Chen ZH, Kim HP and Choi AM: Autophagy in chronic obstructive pulmonary disease: Homeostatic or pathogenic mechanism. Autophagy 5: 235-237, 2009.

12. Li Y, Yu G, Yuan S, Tan C, Lian P, Fu L, Hou Q, Xu B and Wang $\mathrm{H}$ : Cigarette smoke-induced pulmonary inflammation and autophagy are attenuated in Ephx2-deficient mice. Inflammation 40: 497-510, 2017

13. Li Y, Yu G, Yuan S, Tan C, Xie J, Ding Y, Lian P, Fu L, Hou Q, $\mathrm{Xu} \mathrm{B}$ and Wang H: 14,15-Epoxyeicosatrienoic acid suppresses cigarette smoke condensate-induced inflammation in lung epithelial cells by inhibiting autophagy. Am J Physiol Lung Cell Mol Physiol 311: L970-L980, 2016.

14. Pei J, Deng J, Ye Z, Wang J, Gou H, Liu W, Zhao M, Liao M, Yi L and Chen J: Absence of autophagy promotes apoptosis by modulating the ROS-dependent RLR signaling pathway in classical swine fever virus-infected cells. Autophagy 12: 1738-1758, 2016

15. Chen Y, Azad MB and Gibson SB: Methods for detecting autophagy and determining autophagy-induced cell death. Can J Physiol Pharmacol 88: 285-295, 2010.

16. Vestbo J,Hurd SS, Agustí AG, Jones PW, Vogelmeier C, Anzueto A Barnes PJ, Fabbri LM, Martinez FJ, Nishimura M, et al: Global strategy for the diagnosis, management, and prevention of chronic obstructive pulmonary disease: GOLD executive summary. Am J Respir Crit Care Med 187: 347-365, 2013.

17. Furlong HC, Stämpfli MR, Gannon AM and Foster WG: Cigarette smoke exposure triggers the autophagic cascade via activation of the AMPK pathway in mice. Biol Reprod 93: 93, 2015.

18. Gannon AM, Stämpfli MR and Foster WG: Cigarette smoke exposure elicits increased autophagy and dysregulation of mitochondrial dynamics in murine granulosa cells. Biol Reprod 88: 63, 2013.

19. Chen ZH, Wu YF, Wang PL, Wu YP, Li ZY, Zhao Y, Zhou JS, Zhu C, Cao C, Mao YY, et al: Autophagy is essential for ultrafine particle-induced inflammation and mucus hyperproduction in airway epithelium. Autophagy 12: 297-311, 2016.

20. Zhou JS, Zhao Y, Zhou HB, Wang Y, Wu YF, Li ZY, Xuan NX, Zhang C, Hua W, Ying SM, et al: Autophagy plays an essential role in cigarette smoke-induced expression of MUC5AC in airway epithelium. Am J Physiol Lung Cell Mol Physiol 310: L1042-L1052, 2016.

21. Hussain SN and Sandri M: Role of autophagy in COPD skeletal muscle dysfunction. J Appl Physiol (1985) 114: 1273-1281, 2013.

22. Plant PJ, Brooks D, Faughnan M, Bayley T, Bain J, Singer L, Correa J, Pearce D, Binnie M and Batt J: Cellular markers of muscle atrophy in chronic obstructive pulmonary disease. Am J Respir Cell Mol Biol 42: 461-471, 2010.

23. Bodas M and Vij N: Augmenting autophagy for prognosis based intervention of COPD-pathophysiology. Respir Res 18: 83, 2017.

24. Singh K, Matsuyama S, Drazba JA and Almasan A Autophagy-dependent senescence in response to DNA damage and chronic apoptotic stress. Autophagy 8: 236-251, 2012.
25. Bodas M, Patel N, Silverberg D, Walworth K and Vij N: Master autophagy regulator transcription factor EB regulates cigarette smoke-induced autophagy impairment and chronic obstructive pulmonary disease-emphysema pathogenesis. Antioxid Redox Signal 27: 150-167, 2017.

26. Chen ZH, Lam HC, Jin Y, Kim HP, Cao J, Lee SJ, Ifedigbo E, Parameswaran H, Ryter SW and Choi AM: Autophagy protein microtubule-associated protein 1 light chain-3B (LC3B) activates extrinsic apoptosis during cigarette smoke-induced emphysema. Proc Natl Acad Sci USA 107: 18880-18885, 2010.

27. Tsukahara T, Matsuda Y, Usui Y and Haniu H: Highly purified, multi-wall carbon nanotubes induce light-chain 3B expression in human lung cells. Biochem Biophys Res Commun 440: 348-353, 2013.

28. Aparicio IM, Espino J, Bejarano I, Gallardo-Soler A, Campo ML Salido GM, Pariente JA, Peña FJ and Tapia JA: Autophagy-related proteins are functionally active in human spermatozoa and may be involved in the regulation of cell survival and motility. Sci Rep 6: 33647, 2016.

29. Wang Y,Zhu WG and Zhao Y: Autophagy substrate SQSTM1/p62 regulates chromatin ubiquitination during the DNA damage response. Autophagy 13: 212-213, 2017.

30. Bitto A, Lerner CA, Nacarelli T, Crowe E, Torres C and Sell C: P62/SQSTM1 at the interface of aging, autophagy, and disease. Age (Dordr) 36: 9626, 2014

31. Taniguchi K, Yamachika S, He F and Karin M: p62/SQSTM1-Dr. Jekyll and Mr. Hyde that prevents oxidative stress but promotes liver cancer. FEBS Lett 590: 2375-2397, 2016.

32. Schläfli AM, Adams O, Galván JA, Gugger M, Savic S, Bubendorf L, Schmid RA, Becker KF, Tschan MP, Langer R and Berezowska S: Prognostic value of the autophagy markers LC3 and p62/SQSTM1 in early-stage non-small cell lung cancer. Oncotarget 7: 39544-39555, 2016.

33. Hewitt G, Carroll B, Sarallah R, Correia-Melo C, Ogrodnik M, Nelson G, Otten EG, Manni D, Antrobus R, Morgan BA, et al: SQSTM1/p62 mediates crosstalk between autophagy and the UPS in DNA repair. Autophagy 12: 1917-1930, 2016.

34. Kuhn C III, Homer RJ, Zhu Z, Ward N, Flavell RA, Geba GP and Elias JA: Airway hyperresponsiveness and airway obstruction in transgenic mice. Morphologic correlates in mice overexpressing interleukin (IL)-11 and IL-6 in the lung. Am J Respir Cell Mol Biol 22: 289-295, 2000.

35. Bhowmik A, Seemungal TA, Sapsford RJ and Wedzicha JA: Relation of sputum inflammatory markers to symptoms and lung function changes in COPD exacerbations. Thorax 55: 114-120, 2000.

36. Ryter SW, Koo JK and Choi AM: Molecular regulation of autophagy and its implications for metabolic diseases. Curr Opin Clin Nutr Metab Care 17: 329-337, 2014

37. Feizi N, Mehrbod P, Romani B, Soleimanjahi H, Bamdad T, Feizi A, Jazaeri EO, Targhi HS, Saleh M, Jamali A, et al: Autophagy induction regulates influenza virus replication in a time-dependent manner. J Med Microbiol 66: 536-541, 2017.

38. Ma Y, Galluzzi L, Zitvogel L and Kroemer G: Autophagy and cellular immune responses. Immunity 39: 211-227, 2013.

39. Zhong Z, Sanchez-Lopez E and Karin M: Autophagy, NLRP3 inflammasome and auto-inflammatory/immune diseases. Clin Exp Rheumatol 34 (4 Suppl 98): S12-S16, 2016.

40. Vij N, Chandramani-Shivalingappa P and Van Westphal C, Hole R and Bodas M: Cigarette smoke induced autophagyimpairment accelerates lung aging, COPD-emphysema exacerbations and pathogenesis. Am J Physiol Cell Physiol 314: C73-C87, 2018

41. Li D, Hu J, Wang T, Zhang X, Liu L, Wang H, Wu Y, Xu D and Wen F: Silymarin attenuates cigarette smoke extract-induced inflammation via simultaneous inhibition of autophagy and ERK/p38 MAPK pathway in human bronchial epithelial cells. Sci Rep 6: 37751, 2016.

42. Polosukhin VV, Lawson WE, Milstone AP, Egunova SM, Kulipanov AG, Tchuvakin SG, Massion PP and Blackwell TS: Association of progressive structural changes in the bronchial epithelium with subepithelial fibrous remodeling: A potential role for hypoxia. Virchows Arch 451: 793-803, 2007.

43. Baek KJ, Cho JY, Rosenthal P, Alexander LE, Nizet V and Broide DH: Hypoxia potentiates allergen induction of HIF-1 $\alpha$, chemokines, airway inflammation, TGF- $\beta 1$, and airway remodeling in a mouse model. Clin Immunol 147: 27-37, 2013. 